\title{
On Caputo type sequential fractional differential equations with nonlocal integral boundary conditions
}

\author{
Ahmed Alsaedi ${ }^{1}$, Sotiris K Ntouyas ${ }^{1,2}$, Ravi P Agarwal ${ }^{1,3}$ and Bashir Ahmad ${ }^{1 *}$
}

"Correspondence:

bashirahmad_qau@yahoo.com 'Department of Mathematics,

Faculty of Science, King Abdulaziz University, P.O. Box 80203, Jeddah, 21589, Saudi Arabia

Full list of author information is available at the end of the article

\begin{abstract}
This paper investigates a boundary value problem of Caputo type sequential fractional differential equations supplemented with nonlocal Riemann-Liouville fractional integral boundary conditions. Some existence results for the given problem are obtained via standard tools of fixed point theory and are well illustrated with the aid of examples. Some special cases are also presented.

MSC: $34 \mathrm{~A} 08 ; 34 \mathrm{~B} 15$

Keywords: fractional differential equations; sequential fractional derivative; integral boundary conditions; fixed point theorems
\end{abstract}

\section{Introduction}

The subject of fractional calculus has received great attention in the last two decades. Recent work on fractional differential equations supplemented with a variety of initial and boundary conditions clearly reflects an overwhelming interest in the subject; for instance, see [1-12] and the references cited therein. The tools of fractional calculus have considerably improved the mathematical modeling of many real world problems. One of the primary reasons for this development is the nonlocal nature of fractional-order differential operators which can describe the hereditary properties of many important materials. One can find applications of the subject in numerous fields of physical and technical sciences such as biomathematics, blood flow phenomena, ecology, environmental issues, viscoelasticity, aerodynamics, electro-dynamics of complex medium, electrical circuits, electronanalytical chemistry, control theory, etc. For further details, see [13-18]. Some more recent results concerning fractional boundary value problems can be found in a series of papers [19-27].

The concept of sequential fractional derivative is given, for example, on p.209 of the monograph [28]. There is a close connection between the sequential fractional derivatives and the non-sequential Riemann-Liouville derivatives [29, 30]. For some recent work on sequential fractional differential equations, we refer the reader to the papers [31-33]. In $[34,35]$, the authors studied sequential fractional differential equations with different kinds of boundary conditions. Recently, the existence of solutions for higher-order sequential fractional differential inclusions with nonlocal three-point boundary conditions was discussed in [36]. However, to the best of our knowledge, the study of sequential

(c) 2015 Alsaedi et al.: licensee Springer. This is an Open Access article distributed under the terms of the Creative Commons Attribution License (http://creativecommons.org/licenses/by/4.0), which permits unrestricted use, distribution, and reproduction in any medium, provided the original work is properly credited. 
fractional differential equations supplemented with nonlocal Riemann-Liouville type fractional integral boundary conditions has yet to be initiated.

In this paper, we investigate the existence of solutions of a sequential fractional differential equation of the form:

$$
\left({ }^{\mathrm{c}} D^{\alpha}+k^{\mathrm{c}} D^{\alpha-1}\right) x(t)=f(t, x(t)), \quad t \in[0,1], 2<\alpha \leq 3,
$$

supplemented with the boundary conditions

$$
x(0)=0, \quad x^{\prime}(0)=0, \quad x(\zeta)=a \int_{0}^{\eta} \frac{(\eta-s)^{\beta-1}}{\Gamma(\beta)} x(s) d s, \quad \beta>0,
$$

where ${ }^{\mathrm{c}} D^{\alpha}$ denotes the Caputo fractional derivative of order $\alpha, 0<\eta<\zeta<1, f$ is a given continuous function, and $k, a$ are appropriate positive real constants.

Here, we emphasize that the integral boundary condition (1.2) can be understood in the sense that the value of the unknown function at an arbitrary position $\zeta \in(\eta, 1)$ is proportional to the Riemann-Liouville fractional integral of the unknown function $\int_{0}^{\eta} \frac{(\eta-s)^{\beta-1}}{\Gamma(\beta)} x(s) d s$, where $\eta \in(0, \zeta)$. Further, for $\beta=1$, the integral boundary condition reduces to the usual form of a nonlocal integral condition $x(\zeta)=a \int_{0}^{\eta} x(s) d s$.

The contents of the article are arranged as follows. In Section 2, we present a basic result that lays the foundation for defining a fixed point problem equivalent to the given problem (1.1)-(1.2). The main results, based on Banach's contraction mapping principle, Krasnoselskii's fixed point theorem and nonlinear alternative of Leray-Schauder type, are presented in Section 3. The paper concludes with some illustrating examples.

\section{Basic result}

Before presenting an auxiliary lemma, we recall some basic definitions of fractional calculus $[13,14]$.

Definition 2.1 For $(n-1)$-times absolutely continuous function $g:[0, \infty) \rightarrow \mathbb{R}$, the Caputo derivative of fractional order $q$ is defined as

$$
{ }^{\mathrm{c}} D^{q} g(t)=\frac{1}{\Gamma(n-q)} \int_{0}^{t}(t-s)^{n-q-1} g^{(n)}(s) d s, \quad n-1<q<n, n=[q]+1,
$$

where $[q]$ denotes the integer part of the real number $q$.

Definition 2.2 The Riemann-Liouville fractional integral of order $q$ is defined as

$$
I^{q} g(t)=\frac{1}{\Gamma(q)} \int_{0}^{t} \frac{g(s)}{(t-s)^{1-q}} d s, \quad q>0
$$

provided the integral exists.

Definition 2.3 The sequential fractional derivative for a sufficiently smooth function $g(t)$ due to Miller-Ross [28] is defined as

$$
D^{\delta} g(t)=D^{\delta_{1}} D^{\delta_{2}} \cdots D^{\delta_{k}} g(t)
$$

where $\delta=\left(\delta_{1}, \ldots, \delta_{k}\right)$ is a multi-index. 
In general, the operator $D^{\delta}$ in (2.1) can either be Riemann-Liouville or Caputo or any other kind of integro-differential operator. For instance,

$$
{ }^{\mathrm{c}} D^{q} g(t)=D^{-(n-q)}\left(\frac{d}{d t}\right)^{n} g(t), \quad n-1<q<n,
$$

where $D^{-(n-q)}$ is a fractional integral operator of order $n-q$. Here we emphasize that $D^{-p} f(t)=I^{p} f(t), p=n-q$; for more details, see p.87 of [13].

To define the solution for problem (1.1)-(1.2), we establish the following lemma.

Lemma 2.4 For $h \in C([0,1], \mathbb{R})$, the integral solution of the linear equation

$$
\left({ }^{\mathrm{c}} D^{\alpha}+k^{\mathrm{c}} D^{\alpha-1}\right) x(t)=h(t), \quad t \in[0,1], 2<\alpha \leq 3,
$$

supplemented with the boundary conditions (1.2) is given by

$$
\begin{aligned}
x(t)= & \frac{\left(k t-1+e^{-k t}\right)}{\Delta}\left[a \int_{0}^{\eta} \frac{(\eta-s)^{\beta-1}}{\Gamma(\beta)}\right. \\
& \times\left(\int_{0}^{s} e^{-k(s-m)}\left(\int_{0}^{m} \frac{(m-\tau)^{\alpha-2}}{\Gamma(\alpha-1)} h(\tau) d \tau\right) d m\right) d s \\
& \left.-\int_{0}^{\zeta} e^{-k(\zeta-s)}\left(\int_{0}^{s} \frac{(s-\tau)^{\alpha-2}}{\Gamma(\alpha-1)} h(\tau) d \tau\right) d s\right] \\
& +\int_{0}^{t} e^{-k(t-s)}\left(\int_{0}^{s} \frac{(s-\tau)^{\alpha-2}}{\Gamma(\alpha-1)} h(\tau) d \tau\right) d s .
\end{aligned}
$$

Proof Solving (2.2), we obtain

$$
\begin{aligned}
x(t)= & b_{0} e^{-k t}+\frac{b_{1}}{k}\left(1-e^{-k t}\right)+\frac{b_{2}}{k^{2}}\left(k t-1+e^{-k t}\right) \\
& +\int_{0}^{t} e^{-k(t-s)}\left(\int_{0}^{s} \frac{(s-\tau)^{\alpha-2}}{\Gamma(\alpha-1)} h(\tau) d \tau\right) d s,
\end{aligned}
$$

where $b_{0}, b_{1}, b_{2}$ are unknown arbitrary constants. Using the boundary conditions (1.2) in (2.4), we find that $b_{0}=0, b_{1}=0$ and

$$
\begin{aligned}
b_{2}= & \frac{k^{2}}{\Delta}\left[a \int_{0}^{\eta} \frac{(\eta-s)^{\beta-1}}{\Gamma(\beta)}\left(\int_{0}^{s} e^{-k(s-m)}\left(\int_{0}^{m} \frac{(m-\tau)^{\alpha-2}}{\Gamma(\alpha-1)} h(\tau) d \tau\right) d m\right) d s\right. \\
& \left.-\int_{0}^{\zeta} e^{-k(\zeta-s)}\left(\int_{0}^{s} \frac{(s-\tau)^{\alpha-2}}{\Gamma(\alpha-1)} h(\tau) d \tau\right) d s\right],
\end{aligned}
$$

where

$$
\Delta=k \zeta-1+e^{-k \zeta}-\frac{a}{\Gamma(\beta)}\left(\frac{k \eta^{\beta+1}}{\beta(\beta+1)}-\frac{\eta^{\beta}}{\beta}+\int_{0}^{\eta}(\eta-s)^{\beta-1} e^{-k s} d s\right) \neq 0 .
$$

Substituting the values of $b_{0}, b_{1}$ and $b_{2}$ in (2.4) yields the solution (2.3). This completes the proof. 


\section{Existence of solutions}

Let $\mathcal{C}=C([0,1], \mathbb{R})$ denote the Banach space of all continuous functions from $[0,1] \rightarrow \mathbb{R}$ endowed with the sup norm defined by $\|x\|=\sup \{|x(t)|, t \in[0,1]\}<\infty$.

To simplify the proofs in the forthcoming theorems, we establish the bounds for the integrals arising in the sequel.

Lemma 3.1 For $h \in C([0,1], \mathbb{R})$, we have

(i)

$$
\begin{aligned}
& \left|\int_{0}^{\eta} \frac{(\eta-s)^{\beta-1}}{\Gamma(\beta)}\left(\int_{0}^{s} e^{-k(s-m)}\left(\int_{0}^{m} \frac{(m-\tau)^{\alpha-2}}{\Gamma(\alpha-1)} h(\tau) d \tau\right) d m\right) d s\right| \\
& \quad \leq \frac{\eta^{\alpha+\beta-1}}{k^{2} \Gamma(\alpha) \Gamma(\beta)}\left(\eta k+e^{-k \eta}-1\right)\|h\|,
\end{aligned}
$$

(ii) $\left|\int_{0}^{\zeta} e^{-k(\zeta-s)}\left(\int_{0}^{s} \frac{(s-\tau)^{\alpha-2}}{\Gamma(\alpha-1)} h(\tau) d \tau\right) d s\right| \leq \frac{\zeta^{\alpha}}{k \Gamma(\alpha)}\left(1-e^{-k \zeta}\right)\|h\|$,

(iii) $\left|\int_{0}^{t} e^{-k(t-s)}\left(\int_{0}^{s} \frac{(s-\tau)^{\alpha-2}}{\Gamma(\alpha-1)} h(\tau) d \tau\right) d s\right| \leq \frac{1}{k \Gamma(\alpha)}\left(1-e^{-k}\right)\|h\|$.

Proof (i) Obviously,

$$
\int_{0}^{m} \frac{(m-\tau)^{\alpha-2}}{\Gamma(\alpha-1)} d \tau=-\left.\frac{(m-\tau)^{\alpha-1}}{\Gamma(\alpha)}\right|_{0} ^{m}=\frac{m^{\alpha-1}}{\Gamma(\alpha)}
$$

and

$$
\int_{0}^{s} e^{-k(s-m)} \frac{m^{\alpha-1}}{\Gamma(\alpha)} d m \leq \frac{s^{\alpha-1}}{\Gamma(\alpha)} \int_{0}^{s} e^{-k(s-m)} d m=\frac{s^{\alpha-1}}{\Gamma(\alpha)} \frac{\left(1-e^{-k s}\right)}{k} .
$$

Hence

$$
\begin{aligned}
& \left|\int_{0}^{\eta} \frac{(\eta-s)^{\beta-1}}{\Gamma(\beta)}\left(\int_{0}^{s} e^{-k(s-m)}\left(\int_{0}^{m} \frac{(m-\tau)^{\alpha-2}}{\Gamma(\alpha-1)} h(\tau) d \tau\right) d m\right) d s\right| \\
& \quad \leq\|h\| \int_{0}^{\eta} \frac{(\eta-s)^{\beta-1}}{\Gamma(\beta)} \frac{s^{\alpha-1}\left(1-e^{-k s}\right)}{k \Gamma(\alpha)} d s \\
& \quad \leq\|h\| \frac{\eta^{\alpha+\beta-1}}{k \Gamma(\alpha) \Gamma(\beta)} \int_{0}^{\eta}\left(1-e^{-k s}\right) d s=\|h\| \frac{\eta^{\alpha+\beta-1}}{k^{2} \Gamma(\alpha) \Gamma(\beta)}\left(\eta k+e^{-k \eta}-1\right) .
\end{aligned}
$$

The proofs of (ii) and (iii) are similar.

For the sake of convenience, we set

$$
p=\sup _{t \in[0,1]}\left|\frac{\left(k t-1+e^{-k t}\right)}{\Delta}\right|=\frac{1}{|\Delta|}\left(e^{-k}+k-1\right)
$$

and

$$
\Lambda=p\left\{\frac{|a| \eta^{\alpha+\beta-1}}{k^{2} \Gamma(\alpha) \Gamma(\beta)}\left(k \eta+e^{-k \eta}-1\right)+\frac{\zeta^{\alpha-1}\left(1-e^{-k \zeta}\right)}{k \Gamma(\alpha)}\right\}+\frac{1-e^{-k}}{k \Gamma(\alpha)} .
$$

In view of Lemma 2.4, we transform problem (1.1)-(1.2) as

$$
x=\mathcal{S}(x),
$$


where $\mathcal{S}: \mathcal{C} \rightarrow \mathcal{C}$ is defined by

$$
\begin{aligned}
(\mathcal{S} x)(t)= & \frac{\left(k t-1+e^{-k t}\right)}{\Delta}\left[a \int_{0}^{\eta} \frac{(\eta-s)^{\beta-1}}{\Gamma(\beta)}\right. \\
& \times\left(\int_{0}^{s} e^{-k(s-m)}\left(\int_{0}^{m} \frac{(m-\tau)^{\alpha-2}}{\Gamma(\alpha-1)} f(\tau, x(\tau)) d \tau\right) d m\right) d s \\
& \left.-\int_{0}^{\zeta} e^{-k(\zeta-s)}\left(\int_{0}^{s} \frac{(s-\tau)^{\alpha-2}}{\Gamma(\alpha-1)} f(\tau, x(\tau)) d \tau\right) d s\right] \\
& +\int_{0}^{t} e^{-k(t-s)}\left(\int_{0}^{s} \frac{(s-\tau)^{\alpha-2}}{\Gamma(\alpha-1)} f(\tau, x(\tau)) d \tau\right) d s .
\end{aligned}
$$

Observe that problem (1.1)-(1.2) has solutions if the operator equation (3.3) has fixed points.

Theorem 3.2 Let $f:[0,1] \times \mathbb{R} \rightarrow \mathbb{R}$ be a jointly continuous function satisfying the condition

$\left(\mathrm{H}_{1}\right)|f(t, x)-f(t, y)| \leq L|x-y|, \forall t \in[0,1], x, y \in \mathbb{R}$,

where $L$ is the Lipschitz constant. Then the boundary value problem (1.1)-(1.2) has a unique solution if $\Lambda<1 / L$, where $\Lambda$ is given by (3.2).

Proof As the first step, we show that the operator $\mathcal{S}$ given by (3.3) maps $\mathcal{C}$ into itself. For that, we set $\sup _{t \in[0,1]}|f(t, 0)|=M<\infty$. Then, for $x \in \mathcal{C}$, we have

$$
\begin{aligned}
\|(\mathcal{S} x)\|= & \sup _{t \in[0,1]} \mid \frac{\left(k t-1+e^{-k t}\right)}{\Delta}\left[a \int_{0}^{\eta} \frac{(\eta-s)^{\beta-1}}{\Gamma(\beta)}\right. \\
& \times\left(\int_{0}^{s} e^{-k(s-m)}\left(\int_{0}^{m} \frac{(m-x)^{\alpha-2}}{\Gamma(\alpha-1)} f(\tau, x(\tau)) d \tau\right) d m\right) d s \\
& \left.-\int_{0}^{\zeta} e^{-k(\zeta-s)}\left(\int_{0}^{s} \frac{(s-x)^{\alpha-2}}{\Gamma(\alpha-1)} f(\tau, x(\tau)) d \tau\right) d s\right] \\
& +\int_{0}^{t} e^{-k(t-s)}\left(\int_{0}^{s} \frac{(s-x)^{\alpha-2}}{\Gamma(\alpha-1)} f(\tau, x(\tau)) d \tau\right) d s \mid \\
\leq & \sup _{t \in[0,1]} \frac{\left(k t-1+e^{-k t}\right)}{\Delta} \mid\left[| a | \int _ { 0 } ^ { \eta } \frac { ( \eta - s ) ^ { \beta - 1 } } { \Gamma ( \beta ) } \left(\int_{0}^{s} e^{-k(s-m)}\right.\right. \\
& \left.\times\left(\int_{0}^{m} \frac{(m-\tau)^{\alpha-2}}{\Gamma(\alpha-1)}(|f(\tau, x(\tau))-f(\tau, 0)|+|f(\tau, 0)|) d x\right) d m\right) d s \\
& \left.+\int_{0}^{\zeta} e^{-k(\zeta-s)}\left(\int_{0}^{s} \frac{(s-\tau)^{\alpha-2}}{\Gamma(\alpha-1)}(|f(\tau, x(\tau))-f(\tau, 0)|+|f(\tau, 0)|) d x\right) d s\right] \\
& +\int_{0}^{t} e^{-k(t-s)}\left(\int_{0}^{s} \frac{(s-x)^{\alpha-2}}{\Gamma(\alpha-1)}(|f(\tau, x(\tau))-f(\tau, 0)|+|f(\tau, 0)|) d x\right) d s \\
\leq & (L\|x\|+M)\left\{p \left[|a| \int_{0}^{\eta} \frac{(\eta-s)^{\beta-1}}{\Gamma(\beta)}\left(\int_{0}^{s} e^{-k(s-m)}\left(\int_{0}^{m} \frac{(m-\tau)^{\alpha-2}}{\Gamma(\alpha-1)} d \tau\right) d m\right) d s\right.\right. \\
& \left.\left.+\int_{0}^{\zeta} e^{-k(\zeta-s)}\left(\int_{0}^{s} \frac{(s-\tau)^{\alpha-2}}{\Gamma(\alpha-1)} d \tau\right) d s\right]+\int_{0}^{t} e^{-k(t-s)}\left(\int_{0}^{s} \frac{(s-\tau)^{\alpha-2}}{\Gamma(\alpha-1)} d \tau\right) d s\right\}
\end{aligned}
$$




$$
\begin{aligned}
& \leq(L\|x\|+M)\left\{p\left[\frac{|a| \eta^{\alpha+\beta-1}}{k^{2} \Gamma(\alpha) \Gamma(\beta)}\left(k \eta+e^{-k \eta}-1\right)+\frac{\zeta^{\alpha-1}\left(1-e^{-k \zeta}\right)}{k \Gamma(\alpha)}\right]+\frac{1-e^{-k}}{k \Gamma(\alpha)}\right\} \\
& =(L\|x\|+M) \Lambda<\infty .
\end{aligned}
$$

This shows that $\mathcal{S}$ maps $\mathcal{C}$ into itself. Now, for $x, y \in \mathcal{C}$ and for each $t \in[0,1]$, we obtain

$$
\begin{aligned}
\|(\mathcal{S} x)-(\mathcal{S} y)\|= & \sup _{t \in[0,1]}|(\mathcal{S} x)(t)-(\mathcal{S} y)(t)| \\
\leq & \sup _{t \in[0,1]}\left|\frac{\left(k t-1+e^{-k t}\right)}{\Delta}\right|\left[|a| \int_{0}^{\eta} \frac{(\eta-s)^{\beta-1}}{\Gamma(\beta)}\right. \\
& \times\left(\int_{0}^{s} e^{-k(s-m)}\left(\int_{0}^{m} \frac{(m-\tau)^{\alpha-2}}{\Gamma(\alpha-1)}|f(\tau, x(\tau))-f(\tau, y(\tau))| d \tau\right) d m\right) d s \\
& \left.+\int_{0}^{\zeta} e^{-k(\zeta-s)}\left(\int_{0}^{s} \frac{(s-\tau)^{\alpha-2}}{\Gamma(\alpha-1)}|f(\tau, x(\tau))-f(\tau, y(\tau))| d \tau\right) d s\right] \\
& +\int_{0}^{t} e^{-k(t-s)}\left(\int_{0}^{s} \frac{(s-\tau)^{\alpha-2}}{\Gamma(\alpha-1)}|f(\tau, x(\tau))-f(\tau, y(\tau))| d \tau\right) d s \\
\leq & L\|x-y\|\left\{p \left[|a| \int_{0}^{\eta} \frac{(\eta-s)^{\beta-1}}{\Gamma(\beta)}\right.\right. \\
& \times\left(\int_{0}^{s} e^{-k(s-m)}\left(\int_{0}^{m} \frac{(m-x)^{\alpha-2}}{\Gamma(\alpha-1)} d x\right) d m\right) d s \\
& \left.+\int_{0}^{\zeta} e^{-k(\zeta-s)}\left(\int_{0}^{s} \frac{(s-x)^{\alpha-2}}{\Gamma(\alpha-1)} d x\right) d s\right] \\
& \left.+\int_{0}^{t} e^{-k(t-s)}\left(\int_{0}^{s} \frac{(s-x)^{\alpha-2}}{\Gamma(\alpha-1)} d x\right) d s\right\} \\
\leq & L\left\{p\left[\frac{|a| \eta^{\alpha+\beta-1}}{k^{2} \Gamma(\alpha) \Gamma(\beta)}\left(k \eta+e^{-k \eta}-1\right)+\frac{\zeta^{\alpha-1}\left(1-e^{-k \zeta}\right)}{k \Gamma(\alpha)}\right]+\frac{1-e^{-k}}{k \Gamma(\alpha)}\right\}\|x-y\| \\
= & L \Lambda\|x-y\|,
\end{aligned}
$$

where $\Lambda$ is given by (3.2). As $\Lambda<1 / L$, therefore, $\mathcal{S}$ is a contraction. Thus, the conclusion of the theorem follows by the contraction mapping principle. This completes the proof.

Now, we state a known result due to Krasnoselskii [37] which is needed to prove the existence of at least one solution of (1.1)-(1.2).

Theorem 3.3 Let $M$ be a closed, convex, bounded and nonempty subset of a Banach space X. Let $\mathcal{G}_{1}, \mathcal{G}_{2}$ be the operators such that: (i) $\mathcal{G}_{1} x+\mathcal{G}_{2} y \in M$ whenever $x, y \in M$; (ii) $\mathcal{G}_{1}$ is compact and continuous; (iii) $\mathcal{G}_{2}$ is a contraction mapping. Then there exists $z \in M$ such that $z=\mathcal{G}_{1} z+\mathcal{G}_{2} z$.

Theorem 3.4 Assume that $f:[0,1] \times \mathbb{R} \rightarrow \mathbb{R}$ is a jointly continuous function satisfying $\left(\mathrm{H}_{1}\right)$. In addition we suppose that the following assumption holds:

$\left(\mathrm{H}_{2}\right)|f(t, x)| \leq \mu(t), \forall(t, x) \in[0,1] \times \mathbb{R}$ with $\mu \in C([0,1], \mathbb{R})$.

Then the boundary value problem (1.1)-(1.2) has at least one solution on [0,1] if

$$
p\left[\frac{|a| \eta^{\alpha+\beta-1}}{k^{2} \Gamma(\alpha) \Gamma(\beta)}\left(k \eta+e^{-k \eta}-1\right)+\frac{\zeta^{\alpha-1}\left(1-e^{-k \zeta}\right)}{k \Gamma(\alpha)}\right]<1 .
$$


Proof Letting $\sup _{t \in[0,1]}|\mu(t)|=\|\mu\|$, we fix

$$
r \geq \Lambda\|\mu\|
$$

where $\Lambda$ is given by (3.2), and consider $\mathcal{B}_{r}=\{x \in \mathcal{C}:\|x\| \leq r\}$. Define the operators $\mathcal{S}_{1}$ and $\mathcal{S}_{2}$ on $\mathcal{B}_{r}$ as

$$
\begin{aligned}
\left(\mathcal{S}_{1} x\right)(t)= & \int_{0}^{t} e^{-k(t-s)}\left(\int_{0}^{s} \frac{(s-\tau)^{\alpha-2}}{\Gamma(\alpha-1)} f(\tau, x(\tau)) d \tau\right) d s, \\
\left(\mathcal{S}_{2} x\right)(t)= & \frac{\left(k t-1+e^{-k t}\right)}{\Delta}\left[a \int_{0}^{\eta} \frac{(\eta-s)^{\beta-1}}{\Gamma(\beta)}\right. \\
& \times\left(\int_{0}^{s} e^{-k(s-m)}\left(\int_{0}^{m} \frac{(m-\tau)^{\alpha-2}}{\Gamma(\alpha-1)} f(\tau, x(\tau)) d \tau\right) d m\right) d s \\
& \left.-\int_{0}^{\zeta} e^{-k(\zeta-s)}\left(\int_{0}^{s} \frac{(s-\tau)^{\alpha-2}}{\Gamma(\alpha-1)} f(\tau, x(\tau)) d \tau\right) d s\right] .
\end{aligned}
$$

For $x, y \in \mathcal{B}_{r}$, it follows from (3.6) that

$$
\left\|\mathcal{S}_{1} x+\mathcal{S}_{2} y\right\| \leq\left\{p\left[\frac{|a| \eta^{\alpha+\beta-1}}{k^{2} \Gamma(\alpha) \Gamma(\beta)}\left(k \eta+e^{-k \eta}-1\right)+\frac{\zeta^{\alpha-1}\left(1-e^{-k \zeta}\right)}{k \Gamma(\alpha)}\right]+\frac{1-e^{-k}}{k \Gamma(\alpha)}\right\}\|\mu\| \leq r .
$$

Thus, $\mathcal{S}_{1} x+\mathcal{S}_{2} y \in \mathcal{B}_{r}$. In view of condition (3.5), it can easily be shown that $\mathcal{S}_{2}$ is a contraction mapping. The continuity of $f$ implies that the operator $\mathcal{S}_{1}$ is continuous. Also, $\mathcal{S}_{1}$ is uniformly bounded on $\mathcal{B}_{r}$ as

$$
\left\|\mathcal{S}_{1} x\right\| \leq \frac{\left(1-e^{-k}\right)\|\mu\|}{k \Gamma(\alpha)}
$$

Now we prove the compactness of the operator $\mathcal{S}_{1}$. Setting $\Omega=[0,1] \times \mathcal{B}_{r}$, we define $\sup _{(t, x) \in \Omega}|f(t, x)|=M_{r}$, and consequently we get

$$
\begin{aligned}
\left|\left(\mathcal{S}_{1} x\right)\left(t_{1}\right)-\left(\mathcal{S}_{1} x\right)\left(t_{2}\right)\right|= & \mid \int_{0}^{t_{1}} e^{-k\left(t_{1}-s\right)}\left(\int_{0}^{s} \frac{(s-u)^{\alpha-2}}{\Gamma(\alpha-1)} f(u, x(u)) d u\right) d s \\
& -\int_{0}^{t_{2}} e^{-k\left(t_{2}-s\right)}\left(\int_{0}^{s} \frac{(s-u)^{\alpha-2}}{\Gamma(\alpha-1)} f(u, x(u)) d u\right) d s \mid \\
\leq & \frac{M_{r}}{k \Gamma(\alpha)}\left(\left|t_{1}^{\alpha}-t_{2}^{\alpha}\right|+\left|t_{1}^{\alpha} e^{-k t_{1}}-t_{2}^{\alpha} e^{-k t_{2}}\right|\right),
\end{aligned}
$$

which is independent of $x$ and tends to zero as $t_{2} \rightarrow t_{1}$. Thus, $\mathcal{S}_{1}$ is relatively compact on $\mathcal{B}_{r}$. Hence, by the Arzelá-Ascoli theorem, $\mathcal{S}_{1}$ is compact on $\mathcal{B}_{r}$. Thus all the assumptions of Theorem 3.3 are satisfied and the conclusion of Theorem 3.3 implies that the boundary value problem (1.1)-(1.2) has at least one solution on $[0,1]$. This completes the proof.

Remark 3.5 In the above theorem we can interchange the roles of the operators $\mathcal{S}_{1}$ and $\mathcal{S}_{2}$ to obtain the second result replacing (3.5) by the following condition:

$$
\frac{1-e^{-k}}{k \Gamma(\alpha)}<1
$$


In the next theorem we prove the existence of a solution for the boundary value problem (1.1)-(1.2) via Leray-Schauder nonlinear alternative.

Lemma 3.6 (Nonlinear alternative for single-valued maps [38]) Let E be a Banach space, $C$ be a closed, convex subset of $E, U$ be an open subset of $C$ and $0 \in U$. Suppose that $F$ : $\bar{U} \rightarrow C$ is a continuous, compact (that is, $F(\bar{U})$ is a relatively compact subset of $C$ ) map. Then either

(i) F has a fixed point in $\bar{U}$, or

(ii) there is $u \in \partial U$ (the boundary of $U$ in $C$ ) and $\lambda \in(0,1)$ with $u=\lambda F(u$ ).

Theorem 3.7 Suppose that $f:[0,1] \times \mathbb{R} \rightarrow \mathbb{R}$ is a jointly continuous function. Further, it is assumed that the following conditions hold:

$\left(\mathrm{H}_{3}\right)$ There exist a function $\phi \in C\left([0,1], \mathbb{R}^{+}\right)$and a nondecreasing function $\psi: \mathbb{R}^{+} \rightarrow \mathbb{R}^{+}$ such that $|f(t, x)| \leq \phi(t) \psi(\|x\|)$ for all $(t, x) \in[0,1] \times \mathbb{R}$.

$\left(\mathrm{H}_{4}\right)$ There exists a constant $M>0$ such that

$$
\frac{M}{\psi(M)\|\phi\| \Lambda}>1
$$

where $\Lambda$ is given by (3.2).

Then the boundary value problem (1.1)-(1.2) has at least one solution on $[0,1]$.

Proof Consider the operator $\mathcal{S}: \mathcal{C} \rightarrow \mathcal{C}$, where

$$
\begin{aligned}
(\mathcal{S} x)(t)= & \frac{\left(k t-1+e^{-k t}\right)}{\Delta}\left[a \int_{0}^{\eta} \frac{(\eta-s)^{\beta-1}}{\Gamma(\beta)}\right. \\
& \times\left(\int_{0}^{s} e^{-k(s-m)}\left(\int_{0}^{m} \frac{(m-\tau)^{\alpha-2}}{\Gamma(\alpha-1)} f(\tau, x(\tau)) d \tau\right) d m\right) d s \\
& \left.-\int_{0}^{\zeta} e^{-k(\zeta-s)}\left(\int_{0}^{s} \frac{(s-\tau)^{\alpha-2}}{\Gamma(\alpha-1)} f(\tau, x(\tau)) d \tau\right) d s\right] \\
& +\int_{0}^{t} e^{-k(t-s)}\left(\int_{0}^{s} \frac{(s-\tau)^{\alpha-2}}{\Gamma(\alpha-1)} f(\tau, x(\tau)) d \tau\right) d s
\end{aligned}
$$

We show that $\mathcal{S}$ maps bounded sets into bounded sets in $C([0,1], \mathbb{R})$. For a positive number $r$, let $\mathcal{B}_{r}=\{x \in C([0,1], \mathbb{R}):\|x\| \leq r\}$ be a bounded set in $C([0,1], \mathbb{R})$. Then

$$
\begin{aligned}
|(\mathcal{S} x)(t)| \leq & \frac{\left(k t-1+e^{-k t}\right)}{\Delta}\left[a \int_{0}^{\eta} \frac{(\eta-s)^{\beta-1}}{\Gamma(\beta)}\right. \\
& \times\left(\int_{0}^{s} e^{-k(s-m)}\left(\int_{0}^{m} \frac{(m-\tau)^{\alpha-2}}{\Gamma(\alpha-1)} f(\tau, x(\tau)) d \tau\right) d m\right) d s \\
& \left.-\int_{0}^{\zeta} e^{-k(\zeta-s)}\left(\int_{0}^{s} \frac{(s-\tau)^{\alpha-2}}{\Gamma(\alpha-1)} f(\tau, x(\tau)) d \tau\right) d s\right] \\
& +\int_{0}^{t} e^{-k(t-s)}\left(\int_{0}^{s} \frac{(s-\tau)^{\alpha-2}}{\Gamma(\alpha-1)} f(\tau, x(\tau)) d \tau\right) d s \mid \\
\leq & p\left[|a| \int_{0}^{\eta} \frac{(\eta-s)^{\beta-1}}{\Gamma(\beta)}\right.
\end{aligned}
$$




$$
\begin{aligned}
& \times\left(\int_{0}^{s} e^{-k(s-m)}\left(\int_{0}^{m} \frac{(m-\tau)^{\alpha-2}}{\Gamma(\alpha-1)} \phi(\tau) \psi(\|x\|) d \tau\right) d m\right) d s \\
& \left.+\int_{0}^{\zeta} e^{-k(\zeta-s)}\left(\int_{0}^{s} \frac{(s-\tau)^{\alpha-2}}{\Gamma(\alpha-1)} \phi(\tau) \psi(\|x\|) d \tau\right) d s\right] \\
& +\int_{0}^{t} e^{-k(t-s)}\left(\int_{0}^{s} \frac{(s-\tau)^{\alpha-2}}{\Gamma(\alpha-1)} \phi(\tau) \psi(\|x\|) d \tau\right) d s \\
\leq & \psi(\|x\|)\|\phi\|\left\{p\left[\frac{|a| \eta^{\alpha+\beta-1}}{k^{2} \Gamma(\alpha) \Gamma(\beta)}\left(k \eta+e^{-k \eta}-1\right)+\frac{\zeta^{\alpha-1}\left(1-e^{-k \zeta}\right)}{k \Gamma(\alpha)}\right]+\frac{1-e^{-k}}{k \Gamma(\alpha)}\right\} \\
= & \psi(\|x\|)\|\phi\| \Lambda .
\end{aligned}
$$

Consequently,

\section{$\|\mathcal{S} x\| \leq \psi(r)\|\phi\| \Lambda$.}

Next we show that $\mathcal{S}$ maps bounded sets into equicontinuous sets of $C([0,1], \mathbb{R})$. Let $t_{1}, t_{2} \in[0,1]$ with $t_{1}<t_{2}$ and $x \in \mathcal{B}_{r}$, where $\mathcal{B}_{r}$ is a bounded set of $C([0,1], \mathbb{R})$. Then we obtain

$$
\begin{aligned}
\left|(\mathcal{S} x)\left(t_{2}\right)-(\mathcal{S} x)\left(t_{1}\right)\right| \leq & \mid \int_{0}^{t_{1}}\left(e^{-k\left(t_{2}-s\right)}-e^{-k\left(t_{1}-s\right)}\right)\left(\int_{0}^{s} \frac{(s-u)^{\alpha-2}}{\Gamma(\alpha-1)} f(u, x(u)) d u\right) d s \\
& +\int_{t_{1}}^{t_{2}} e^{-k\left(t_{2}-s\right)}\left(\int_{0}^{s} \frac{(s-u)^{\alpha-2}}{\Gamma(\alpha-1)} f(u, x(u)) d u\right) d s \mid \\
& +\mid \frac{k\left(t_{2}-t_{1}\right)+e^{-k t_{2}}-e^{-k t_{1}}}{\Delta}\left[a \int_{0}^{\eta} \frac{(\eta-s)^{\beta-1}}{\Gamma(\beta)}\right. \\
& \times\left(\int_{0}^{s} e^{-k(s-m)}\left(\int_{0}^{m} \frac{(m-\tau)^{\alpha-2}}{\Gamma(\alpha-1)} f(\tau, x(\tau)) d \tau\right) d m\right) d s \\
& \left.-\int_{0}^{\zeta} e^{-k(\zeta-s)}\left(\int_{0}^{s} \frac{(s-\tau)^{\alpha-2}}{\Gamma(\alpha-1)} f(\tau, x(\tau)) d \tau\right) d s\right] \mid \\
\leq & \mid \int_{0}^{t_{1}}\left(e^{-k\left(t_{2}-s\right)}-e^{-k\left(t_{1}-s\right)}\right)\left(\int_{0}^{s} \frac{(s-u)^{\alpha-2}}{\Gamma(\alpha-1)} \psi(r) \phi(u) d u\right) d s \\
& +\int_{t_{1}}^{t_{2}} e^{-k\left(t_{2}-s\right)}\left(\int_{0}^{s} \frac{(s-u)^{\alpha-2}}{\Gamma(\alpha-1)} \psi(r) \phi(u) d u\right) d s \mid \\
& +\mid \frac{k\left(t_{2}-t_{1}\right)+e^{-k t_{2}}-e^{-k t_{1}}}{\Delta}\left[a \int_{0}^{\eta} \frac{(\eta-s)^{\beta-1}}{\Gamma(\beta)}\right. \\
& \times\left(\int_{0}^{s} e^{-k(s-m)}\left(\int_{0}^{m} \frac{(m-\tau)^{\alpha-2}}{\Gamma(\alpha-1)} \psi(r) \phi(\tau) d \tau\right) d m\right) d s \\
& \left.-\int_{0}^{\zeta} e^{-k(\zeta-s)}\left(\int_{0}^{s} \frac{(s-\tau)^{\alpha-2}}{\Gamma(\alpha-1)} \psi(r) \phi(\tau) d \tau\right) d s\right] .
\end{aligned}
$$

Obviously, the right-hand side of the above inequality tends to zero independently of $x \in$ $\mathcal{B}_{r}$ as $t_{2}-t_{1} \rightarrow 0$. As $\mathcal{S}$ satisfies the above assumptions, therefore it follows by the ArzeláAscoli theorem that $\mathcal{S}: \mathcal{C} \rightarrow \mathcal{C}$ is completely continuous.

The result will follow from the Leray-Schauder nonlinear alternative (Lemma 3.6) once we have proved the boundedness of the set of all solutions to equations $x=\lambda \mathcal{S} x$ for $\lambda \in$ $[0,1]$. 
Let $x$ be a solution. Then, for $t \in[0,1]$, and using the computations employed in proving that $\mathcal{S}$ is bounded, we have

$$
\begin{aligned}
|x(t)| & =|\lambda(\mathcal{S} x)(t)| \\
& \leq \psi(\|x\|)\|\phi\|\left\{p\left[\frac{|a| \eta^{\alpha+\beta-1}}{k^{2} \Gamma(\alpha) \Gamma(\beta)}\left(k \eta+e^{-k \eta}-1\right)+\frac{\zeta^{\alpha-1}\left(1-e^{-k \zeta}\right)}{k \Gamma(\alpha)}\right]+\frac{1-e^{-k}}{k \Gamma(\alpha)}\right\} \\
& =\psi(\|x\|)\|\phi\| \Lambda .
\end{aligned}
$$

Consequently, we have

$$
\frac{\|x\|}{\psi(\|x\|)\|\phi\| \Lambda} \leq 1
$$

In view of $\left(\mathrm{H}_{4}\right)$, there exists $M$ such that $\|x\| \neq M$. Let us set

$$
U=\{x \in C([0,1], \mathbb{R}):\|x\|<M\} .
$$

Note that the operator $\mathcal{S}: \bar{U} \rightarrow C([0,1], \mathbb{R})$ is continuous and completely continuous. From the choice of $U$, there is no $x \in \partial U$ such that $x=\lambda \mathcal{S}(x)$ for some $\lambda \in(0,1)$. Consequently, by the nonlinear alternative of Leray-Schauder type (Lemma 3.6), we deduce that $\mathcal{S}$ has a fixed point $x \in \bar{U}$ which is a solution of problem (1.1)-(1.2). This completes the proof.

\section{Examples}

The following example is concerned with the illustration of Theorem 3.2.

Example 4.1 Consider the problem

$$
\left\{\begin{array}{l}
{ }^{\mathrm{c}} D^{3 / 2}(D+2) x(t)=\frac{L}{2}\left(\sqrt{t^{2}+1}+\sin t+x(t)+\tan ^{-1} x(t)\right), \quad 0 \leq t \leq 1, \\
x(0)=0, \quad x^{\prime}(0)=0, \quad x(1 / 2)=\int_{0}^{1 / 3} x(s) d s
\end{array}\right.
$$

Here, $\alpha=5 / 2, f(t, x(t))=\frac{L}{2}\left(\sqrt{t^{2}+1}+\sin t+x(t)+\tan ^{-1} x(t)\right), k=2, a=1, \eta=1 / 3, \zeta=1 / 2$, $\beta=1$. Clearly

$$
|f(t, x)-f(t, y)| \leq L\left|x-y+\tan ^{-1} x-\tan ^{-1} y\right| / 2 \leq L|x-y| .
$$

With the given values, we find that $\Delta \approx 0.346810, p \approx 3.273652, \Lambda \approx 0.607518$. For $L<$ $1 / \Lambda \approx 1.646042$, it follows by Theorem 3.2 that problem (4.1) has a unique solution.

Next, we discuss an illustrative example for Theorem 3.7.

Example 4.2 Let us consider problem (4.1) with $f(t, x(t))=\frac{e^{-t}}{4 \sqrt{1+t^{2}}}\left(x^{2}+1\right)$.

We check the conditions of Theorem 3.7. Clearly assumption $\left(\mathrm{H}_{3}\right)$ holds with $\|\phi\|=$ $1 / 4, \psi(\|x\|)=1+\|x\|^{2}$; and by assumption $\left(\mathrm{H}_{4}\right)$ we find that $M_{1}<M<M_{2}$, where $M_{1} \approx$ 0.155555 and $M_{2} \approx 6.4286125$. Thus the conclusion of Theorem 3.7 applies, and hence problem (4.1) with the given value of $f(t, x(t))$ has a solution on $[0,1]$. 


\section{Conclusions}

We have obtained some existence results for a boundary value problem of Caputo type sequential fractional differential equations with nonlocal Riemann-Liouville fractional integral boundary conditions with the aid of Banach's contraction mapping principle, Krasnoselskii's fixed point theorem and nonlinear alternative of Leray-Schauder type. We observe that some new special results follow by fixing the parameters involved in the given problem. For instance, if we choose $\beta=1$, then the results of this paper correspond to a Caputo type sequential fractional differential equation with the boundary conditions of the form $x(0)=0, x^{\prime}(0)=0, x(\zeta)=a \int_{0}^{\eta} x(s) d s$. Further, in the limit $a \rightarrow 0^{+}$, we obtain the results for the boundary conditions $x(0)=0, x^{\prime}(0)=0, x(\zeta)=0$.

\section{Competing interests}

The authors declare that they have no competing interests.

\section{Authors' contributions}

Each of the authors, AA, SKN, RPA and BA, contributed to each part of this work equally and read and approved the final version of the manuscript.

\section{Author details}

${ }^{1}$ Department of Mathematics, Faculty of Science, King Abdulaziz University, P.O. Box 80203, Jeddah, 21589, Saudi Arabia. ${ }^{2}$ Department of Mathematics, University of loannina, loannina, 451 10, Greece. ${ }^{3}$ Department of Mathematics, Texas A\&M University-Kingsville, Kingsville, TX 78363-8202, USA.

\section{Acknowledgements}

This article was funded by the Deanship of Scientific Research (DSR), King Abdulaziz University, Jeddah, Saudi Arabia. The authors, therefore, acknowledge technical and financial support of KAU. The authors also thank the reviewers for their useful comments.

Received: 20 November 2014 Accepted: 19 January 2015 Published online: 31 January 2015

\section{References}

1. Ahmad, B: Existence of solutions for irregular boundary value problems of nonlinear fractional differential equations. Appl. Math. Lett. 23, 390-394 (2010)

2. Ahmad, B, Nieto, JJ: Riemann-Liouville fractional integro-differential equations with fractional nonlocal integral boundary conditions. Bound. Value Probl. 2011, 36 (2011)

3. Liang, S, Zhang, J: Existence of multiple positive solutions for $m$-point fractional boundary value problems on an infinite interval. Math. Comput. Model. 54, 1334-1346 (2011)

4. Su, X: Solutions to boundary value problem of fractional order on unbounded domains in a Banach space. Nonlinear Anal. 74, 2844-2852 (2011)

5. Bai, ZB, Sun, W: Existence and multiplicity of positive solutions for singular fractional boundary value problems. Comput. Math. Appl. 63, 1369-1381 (2012)

6. Agarwal, RP, O'Regan, D, Stanek, S: Positive solutions for mixed problems of singular fractional differential equations Math. Nachr. 285, 27-41 (2012)

7. Cabada, A, Wang, G: Positive solutions of nonlinear fractional differential equations with integral boundary value conditions. J. Math. Anal. Appl. 389, 403-411 (2012)

8. Ahmad, B, Ntouyas, SK, Alsaedi, A: A study of nonlinear fractional differential equations of arbitrary order with Riemann-Liouville type multistrip boundary conditions. Math. Probl. Eng. 2013, Article ID 320415 (2013)

9. Zhang, L, Wang, G, Ahmad, B, Agarwal, RP: Nonlinear fractional integro-differential equations on unbounded domains in a Banach space. J. Comput. Appl. Math. 249, 51-56 (2013)

10. Ahmad, B, Ntouyas, SK: Existence results for higher order fractional differential inclusions with multi-strip fractional integral boundary conditions. Electron. J. Qual. Theory Differ. Equ. 2013, 20 (2013)

11. O'Regan, D, Stanek, S: Fractional boundary value problems with singularities in space variables. Nonlinear Dyn. 71, 641-652 (2013)

12. Baleanu, D, Mustafa, OG, O'Regan, D: A uniqueness criterion for fractional differential equations with Caputo derivative. Nonlinear Dyn. 71, 635-640 (2013)

13. Podlubny, I: Fractional Differential Equations. Academic Press, San Diego (1999)

14. Kilbas, AA, Srivastava, HM, Trujillo, JJ: Theory and Applications of Fractional Differential Equations. North-Holland Mathematics Studies, vol. 204. Elsevier, Amsterdam (2006)

15. Sabatier, J, Agrawal, OP, Machado, JAT (eds.): Advances in Fractional Calculus: Theoretical Developments and Applications in Physics and Engineering. Springer, Dordrecht (2007)

16. Tomovski, Z, Hilfer, R, Srivastava, HM: Fractional and operational calculus with generalized fractional derivative operators and Mittag-Leffler type functions. Integral Transforms Spec. Funct. 21, 797-814 (2010)

17. Konjik, S, Oparnica, L, Zorica, D: Waves in viscoelastic media described by a linear fractional model. Integral Transforms Spec. Funct. 22, 283-291 (2011)

18. Keyantuo, $\mathrm{V}$, Lizama, C: A characterization of periodic solutions for time-fractional differential equations in UMD spaces and applications. Math. Nachr. 284, 494-506 (2011) 
19. Graef, JR, Kong, L, Wang, M: Existence and uniqueness of solutions for a fractional boundary value problem on a graph. Fract. Calc. Appl. Anal. 17, 499-510 (2014)

20. Wang, G, Liu, S, Zhang, L: Eigenvalue problem for nonlinear fractional differential equations with integral boundary conditions. Abstr. Appl. Anal. 2014, Article ID 916260 (2014)

21. Ahmad, B, Agarwal, RP: Some new versions of fractional boundary value problems with slit-strips conditions. Bound. Value Probl. 2014, 175 (2014)

22. Gambo, Y, Jarad, F, Baleanu, D, Abdeljawad, T: On Caputo modification of the Hadamard fractional derivatives. Adv. Differ. Equ. 2014, 10 (2014)

23. Baleanu, D, Nazemi, SZ, Rezapour, S: Attractivity for a k-dimensional system of fractional functional differential equations and global attractivity for a $k$-dimensional system of nonlinear fractional differential equations. J. Inequal. Appl. 2014, 31 (2014)

24. Ahmad, B, Ntouyas, SK: Initial-value problems for hybrid Hadamard fractional differential equations. Electron. J. Differ. Equ. 2014, 161 (2014)

25. Tariboon, J, Sitthiwirattham, T, Ntouyas, SK: Existence results for fractional differential inclusions with multi-point and fractional integral boundary conditions. J. Comput. Anal. Appl. 17, 343-360 (2014)

26. Zhang, L, Ahmad, B, Wang, G: Successive iterations for positive extremal solutions of nonlinear fractional differential equations on a half line. Bull. Aust. Math. Soc. 91, 116-128 (2015)

27. Ahmad, B, Ntouyas, SK: Nonlocal fractional boundary value problems with slit-strips boundary conditions. Fract. Calc. Appl. Anal. 18, 261-280 (2015)

28. Miller, KS, Ross, B: An Introduction to the Fractional Calculus and Fractional Differential Equations. Wiley, New York (1993)

29. Wei, Z, Dong, W: Periodic boundary value problems for Riemann-Liouville sequential fractional differential equations. Electron. J. Qual. Theory Differ. Equ. 2011, 87 (2011)

30. Wei, Z, Li, Q, Che, J: Initial value problems for fractional differential equations involving Riemann-Liouville sequential fractional derivative. J. Math. Anal. Appl. 367, 260-272 (2010)

31. Klimek, M: Sequential fractional differential equations with Hadamard derivative. Commun. Nonlinear Sci. Numer. Simul. 16, 4689-4697 (2011)

32. Baleanu, D, Mustafa, OG, Agarwal, RP: On $\mathrm{L}^{p}$-solutions for a class of sequential fractional differential equations. Appl. Math. Comput. 218, 2074-2081 (2011)

33. Bai, C: Impulsive periodic boundary value problems for fractional differential equation involving Riemann-Liouville sequential fractional derivative. J. Math. Anal. Appl. 384, 211-231 (2011)

34. Ahmad, B, Nieto, JJ: Sequential fractional differential equations with three-point boundary conditions. Comput. Math Appl. 64, 3046-3052 (2012)

35. Ahmad, B, Nieto, JJ: Boundary value problems for a class of sequential integrodifferential equations of fractional order. J. Funct. Spaces Appl. 2013, Article ID 149659 (2013)

36. Ahmad, B, Ntouyas, SK: On higher-order sequential fractional differential inclusions with nonlocal three-point boundary conditions. Abstr. Appl. Anal. 2014, Article ID 659405 (2014)

37. Krasnoselskii, MA: Two remarks on the method of successive approximations. Usp. Mat. Nauk 10, 123-127 (1955)

38. Granas, A, Dugundji, J: Fixed Point Theory. Springer, New York (2003)

\section{Submit your manuscript to a SpringerOpen ${ }^{\odot}$ journal and benefit from:}

- Convenient online submission

- Rigorous peer review

Immediate publication on acceptance

- Open access: articles freely available online

- High visibility within the field

- Retaining the copyright to your article 